\title{
Adherence and Perception of Care Among Patients with Multidrug Resistant Tuberculosis in North Central Nigeria
}

\author{
Laura Madukaji ${ }^{1, ~ *, ~ E b e n e z e r ~ O b i ~ D a n i e l ~}{ }^{1}$, Francis Ejeh ${ }^{2}$, Adewole Olanisun Olufemi $^{3}$, \\ Ahmed Mamuda Bello ${ }^{1}$, Paul Olaiya Abiodun ${ }^{1}$, Israel Olukayode Popoola ${ }^{4}$, Kabir Yunusa Amari ${ }^{5}$, \\ Christiana Asibi Ogben ${ }^{1}$, Michael Oladapo Olagbegi ${ }^{6}$, Gabriel Omoniyi Ayeni ${ }^{1}$, \\ Olayinka Victor Ojo ${ }^{1}$, John Danjuma Mawak ${ }^{7}$
}

\author{
${ }^{1}$ Department of Public Health, Texila American University, Georgetown, Guyana \\ ${ }^{2}$ Department of Veterinary Microbiology, University of Maiduguri, Borno, Nigeria \\ ${ }^{3}$ Department of Medicine, Obafemi Awolowo University, Ile Ife, Nigeria \\ ${ }^{4}$ Department of Epidemiology and Community Health, University of Ilorin, Ilorin, Nigeria \\ ${ }^{5}$ Department of Psychology, Benue State University, Makurdi, Nigeria \\ ${ }^{6}$ South African National Bioinformatics Institute, University of the Western Cape, Western Cape, South Africa \\ ${ }^{7}$ Department of Microbiology, University of Jos, Plateau, Nigeria
}

Email address:

lauradonbabe@yahoo.com (L. Madukaji)

${ }^{*}$ Corresponding author

\section{To cite this article:}

Laura Madukaji, Ebenezer Obi Daniel, Francis Ejeh, Adewole Olanisun Olufemi, Ahmed Mamuda Bello, Paul Olaiya Abiodun, Israel Olukayode Popoola, Kabir Yunusa Amari, Christiana Asibi Ogben, Michael Oladapo Olagbegi, Gabriel Omoniyi Ayeni, Olayinka Victor Ojo, John Danjuma Mawak. Adherence and Perception of Care Among Patients with Multidrug Resistant Tuberculosis in North Central Nigeria. International Journal of Infectious Diseases and Therapy. Vol. 6, No. 3, 2021, pp. 90-96. doi: 10.11648/j.ijidt.20210603.12

Received: July 19, 2021; Accepted: July 28, 2021; Published: August 4, 2021

\begin{abstract}
The efforts geared towards control of Tuberculosis is threatened by the spread of multidrug resistance. Severe forms of multidrug resistant TB termed Pre-extensive (Pre-XDR) and Extensively Drug resistant (XDR) TB have been reported by different countries including Indian, China, Russia and Nigeria. Previous studies have shown a strong association between these various forms of resistance and non-adherence. The study was conducted to identify the factors affecting adherence, assess level of adherence and the perception of care among Multidrug resistant clients in North Central Nigeria. Specific \& structures Questionnaires were administered to 96 MDR TB patients and 67 healthcare providers (representing $50 \%$ of each of the professional group). Few patients and healthcare providers were later selected from the number that responded to questionnaires and were involved in Focus Group Discussion. Responses from participants showed that the factors affecting adherence were alcohol addiction $(72.9 \%)$, lack of social support $(88.5 \%)$, feeling cured $(28.1 \%)$, access to treatment centre $(46.9 \%)$, non-payment \& inadequate incentives/enablers for patients to continue treatment (38.5\%), Stigma and discrimination (31.3\%), financial difficulty (25\%), treatment side effect (39.6\%) and long treatment course \& high pill burden (55.2\%). Patients' level of satisfaction on care was high (66.7\%) indicating a satisfactory perception. This study revealed that factors affecting non-adherence were multifactorial and patients' perception on MDR TB care was satisfactory.
\end{abstract}

Keywords: Adherence, Perception of MDR TB Care, Multidrug Resistant Tuberculosis, North Central, Nigeria

\section{Introduction}

The efforts geared towards control of Tuberculosis (TB) and Multidrug Resistant Tuberculosis (MDR TB) is threatened by the spread of multidrug resistance including its severe forms. Increasing rate of Pre-XDR and XDR TB which are severe forms of MDR TB have been reported at 65 countries. Nigeria is among the 30 high burden countries for 
TB, MDR TB and TB-HIV. [26]. High prevalence of Pre-XDR TB among the multidrug resistant TB cases has been reported in Nigeria [4].

According to previous studies carried out, development of Multidrug resistant TB has been majorly linked to treatment non-adherence and loss to follow up. [25, 16, 29]. Despite many national and international efforts made in preventing and controlling TB as well as implementing DOT in almost all parts of WHO regions, patients still fail to complete their treatment to be declared. [17, 18]. Reports show that a considerable number of TB cases fail after several treatments, many relapse after completion of the treatment, many undergo retreatment after completion of treatment, and many developed MDR-TB among retreatment cases (20\%) throughout the world [19].

Severe forms of multidrug resistant TB termed Pre-extensive (Pre-XDR) and Extensively Drug resistant (XDR) TB have been reported by different countries. Previous studies have reported high prevalence of Multidrug resistant Tuberculosis and factors responsible for the increase in the prevalence of MDR TB in Ethiopia, Indian, China and Russia. [20]. They found a strong association between these various forms of resistance and multiple incomplete treatment regimens, non-adherence, poor infection control practices, and mutation of the resistant TB strains. Nigeria is not excluded from the list of countries that have reported high prevalence of MDR TB and Pre-XDR TB [4]. In South Africa, medication adherence was found to be significantly higher for antiretroviral than for TB medications due to the long treatment course for MDR TB patients as well as adverse drug reaction [22]. In South Western zone of Nigeria, the Prevalence of Pre-XDR TB was reported to be as high as $16.7 \%$. It was assumed that abuse of fluoroquinolone (one of the second line anti-TB drugs) could be the reason for the high frequency of fluoroquinolone resistance observed in the study.

Treatment adherence is considered a primary determinant and a proxy for treatment success. Treatment failure, increase mortality, development and spread of drug resistance, prolonged infectivity are possible poor health outcome of non-adherence to TB therapy and this pose a serious health risk for individuals and communities [21]. Non-adherence to treatment has been reported to be a major factor in development of drug resistant tuberculosis which is due to patient, health worker and health system factors. Major challenges include poor provider-patient communication, inadequate knowledge about a drug and its use, not being convinced of the need for treatment, fear of adverse effects of the drug, long term drug regimens, complex regimens that require numerous medications with varying dosing schedules.

The specific aim of this research was to identify factors responsible for non-adherence and assess perception of care and services among Multidrug resistant clients. Addressing these factors is important because of the role they play in determining treatment adherence. Prioritizing patients through listening to them and treating them as ultimate stakeholders in treatment program would help to develop consistent adherence, retention of patients and reduction of the prevalence of MDR-TB cases. A better understanding of these factors would inform development of interventions to strengthen a patient-centered approach for the delivery of TB programs and services. In North Central Nigeria, no such study has been conducted.

\section{Method}

\subsection{Study Setting}

This was a qualitative study conducted at Benue, Kaduna and Plateau States among Multidrug Resistant TB patients enrolled into treatment at community and facility-based treatment centres. The 3 states where this study was conducted are grouped under the North Central Zone. Benue State has a population of 4,253,641 with 23 Local Government Areas (LGAs). Plateau State has estimated population of about 3.5 million with 17 LGA, Kaduna has a population of 6,113,503 with 23 LGA. Each State has TB and Leprosy Control Program being overseen by the National TB and Leprosy Control Program. National Tuberculosis and Leprosy Control Programme (NTBLCP) adopted a community-based treatment approach to the management of Drug Resistant Tuberculosis (DR TB) to complement facility or hospital-based treatment approach. DR TB data from communities and facilities are collated by DR TB focal person in each state and reported to the State TB and Leprosy Control Program Manager, who in turn report to the NTBLCP. There are 3 major facility-based treatment centres in the 3 states used for the study which are $\mathrm{St}$ Vincent Hospital Aliade, Benue; National TB and Leprosy Training Centre (NTBLTC) Saye, Kaduna and Jos University Teaching Hospital, Plateau.

\subsection{Data Collection}

Three Questionnaires were designed for patients, healthcare providers at the treatment centre and healthcare providers at the States TB Program. Collection of data took place from July 2020 to December 2020. The questionnaires were piloted among forty-five patients and thirty healthcare providers based on questionnaire pilot sampling of Lackey and Wingate [23]. This was to refine the questionnaire to ensure clarity, understanding, and acceptability. Questionnaires were reviewed before data collection for the study commenced. The questionnaires were administered to participants by trained data collectors and Focus Group Discussion conducted. Focus Group Discussion was conducted in English and dialects of participants. The discussions had in participants' dialect were audio recorded and later translated to English.

\subsection{Study Participants}

Primary target population were Multidrug resistant TB patients at Drug Resistant TB treatment centres while the secondary target population were the healthcare providers. The minimum sample size for the number of participants who were interviewed was calculated using EPI INFO version 7 


\section{StatCalc [24].}

\subsection{Ethical Considerations}

Ethical approval for the study was obtained from the States' Ministry of Health Research Ethical Committee. Permission to conduct the study at the facilities was granted by the institutions. All participants gave their informed and written consent before participating in the study, and their anonymity, privacy and confidentiality was respected.

\subsection{Data Analysis}

Data were entered in Microsoft Excel, coded and later imported into IBM SPSS (Version 16) for statistical analysis. Descriptive statistics were used to analyze categorical variables from respondent's socio-demographic and clinical characteristics and were presented as frequencies and percentages in tables and charts. Inferential statistics were applied depending upon the nature of data and variable.

\section{Results}

Table 1. Demographic Characteristics of study participants for assessment of adherence factors.

\begin{tabular}{|c|c|c|c|c|c|}
\hline Patients & & & Healthcare workers & & \\
\hline Variable & Frequency & Percentage & Variable & Frequency & Percentage \\
\hline Age (mean $33.57 \pm 0.5$ ) & & & Age $(32.78 \pm 0.5)$ & & \\
\hline Sex & & & Sex & & \\
\hline Male & 53 & 55.2 & Male & 32 & 48 \\
\hline Female & 43 & 44.8 & Female & 35 & 52 \\
\hline Marital status & & & Marital status & & \\
\hline Single & 25 & 26 & Single & 10 & 15 \\
\hline Married & 63 & 65.6 & Married & 57 & 85 \\
\hline Divorced & 5 & 5.2 & & & \\
\hline Widowed & 3 & 3.1 & & & \\
\hline \multicolumn{6}{|l|}{ Separated } \\
\hline Religious affiliation & & & Years of experience in TB & & \\
\hline Muslim & 45 & 46.9 & $<1$ year & 4 & 6 \\
\hline Pentecostal & 16 & 16.7 & $1-3$ years & 21 & 31.4 \\
\hline Catholic & 31 & 32.3 & 4-6 years & 29 & 43.2 \\
\hline Orthodox & 4 & 4.2 & 7-10 years & 12 & 18 \\
\hline Residency & & & $>10$ years & 1 & 1.5 \\
\hline Urban & 69 & 71.9 & & & \\
\hline Rural & 27 & 28.1 & & & \\
\hline \multicolumn{6}{|l|}{ Education } \\
\hline No formal education & 5 & 5.2 & & & \\
\hline Able to read \& write & 9 & 9.4 & & & \\
\hline Primary & 18 & 18.8 & & & \\
\hline Secondary & 47 & 49 & & & \\
\hline Higher Institution & 17 & 17.7 & & & \\
\hline Profession & & & Profession & & \\
\hline Student & 15 & 15.6 & Doctors & 11 & 16.4 \\
\hline Farmer & 23 & 24.0 & Nurses & 19 & 28.4 \\
\hline Housewife & 3 & 3.1 & Pharmacist & 5 & 7.5 \\
\hline Petty trading & 21 & 21.9 & M \& E Officer & 9 & 13.4 \\
\hline Unemployed & 22 & 22.9 & Lab Scientist & 7 & 10.4 \\
\hline Public servant & 5 & 5.2 & CHEWs & 6 & 9 \\
\hline Civil servant & 1 & 1.0 & Others & 10 & 14.9 \\
\hline Others & 6 & 6.3 & & & \\
\hline
\end{tabular}

Table 2. Common factors affecting adherence reported through FGDs \& questionnaire.

\begin{tabular}{ll}
\hline Patient related & Alcohol addiction, lack of social support, non challant behavior of not taking drugs as symptoms have been relieved \\
Healthcare Provider related & Treatment centre access related, Inadequate enablers for patients to continue treatment, Lack of social support programs \\
Socio-economic status & Stigma \& discrimination, Financial difficulty, unable to take care of comorbidities due to financial constraint \\
Medication related & Side effect of treatment, long treatment duration \& high pill burden \\
\hline
\end{tabular}


Table 3. Reasons for non-adherence to MDR TB treatment.

\begin{tabular}{|c|c|c|c|c|c|c|}
\hline \multirow{2}{*}{ Variable } & \multicolumn{3}{|c|}{ Frequency } & \multicolumn{3}{|c|}{ Percentage } \\
\hline & Yes & No & Partial & Yes & No & Partial \\
\hline Available caregiver at home & 85 & 11 & 0 & 88.5 & 11.5 & 0 \\
\hline Knowledge about progress of treatment & 81 & 14 & 1 & 84.4 & 14.6 & 1 \\
\hline Treatment is no more necessary as I am old & 4 & 92 & - & 4.2 & 95.8 & - \\
\hline Treatment course is too long $\&$ dose too large & 53 & 43 & - & 55.2 & 44.8 & - \\
\hline Caregiver does not really support & 13 & 83 & - & 13.5 & 86.5 & - \\
\hline Cannot cope with the side effects of the drugs & 38 & 58 & - & 39.6 & 60.4 & - \\
\hline Comorbidity makes me interrupt treatment & 25 & 71 & - & 26 & 74 & - \\
\hline Strict adherence to Doctor's advise & 79 & 14 & - & 84.9 & 15.1 & - \\
\hline Not satisfied with healthcare provider's services & 8 & 85 & - & 8.3 & 88.5 & - \\
\hline Financial difficulty & 24 & 72 & - & 25 & 75 & - \\
\hline Long distance to treatment centre & 45 & 51 & - & 46.9 & 53.1 & - \\
\hline
\end{tabular}

Tables 2 and 3 are summaries of the factors that affect adherence and treatment outcome. Healthcare workers identified alcohol addiction as a factor negatively affecting treatment adherence. Healthcare provider reported that the alcohol abuse makes the patients to be stubborn, not to understand information during counselling and therefore makes them not to adhere to instructions. Other factors identified were lack of support (88.5\%), feeling cured (28.1\%), access to treatment centre $(46.9 \%)$, nonpayment \& inadequate incentives/enablers for patients to continue treatment (38.5\%), Stigma and discrimination (31.3\%), financial difficulty (25\%), treatment side effect (39.6\%) and long treatment course \& high pill burden (55.2\%).

Table 4. Responses to Patient-Healthcare Provider relationship.

\begin{tabular}{|c|c|c|c|c|c|c|}
\hline \multirow{2}{*}{ Variable } & \multicolumn{3}{|c|}{ Frequency } & \multicolumn{3}{|c|}{ Percentage } \\
\hline & Yes & No & Partial & Yes & No & Partial \\
\hline Feeling at home when attended to by HCW & 78 & 15 & 3 & 81.3 & 15.6 & 3.1 \\
\hline Feeling stigmatized & 30 & 64 & 2 & 31.3 & 66.7 & 2.1 \\
\hline Feeling your privacy is respected & 74 & 12 & 10 & 77.1 & 12.5 & 10.4 \\
\hline Incentives or enablers to continue treatment & 54 & 37 & 5 & 56.3 & 38.5 & 5.2 \\
\hline Receive counselling \& health information & 89 & 5 & 2 & 92.7 & 5.2 & 2.1 \\
\hline Understand the health information & 85 & 2 & 9 & 88.5 & 2.1 & 9.4 \\
\hline Do you think all information shared are kept confidential? & 82 & 3 & 11 & 85.4 & 3.1 & 11.5 \\
\hline Waiting time too long? & 36 & 50 & 10 & 37.5 & 52.1 & 10.4 \\
\hline Treatment preference at treatment centre or home? & 57 & 26 & 13 & 59.4 & 27.1 & 13.5 \\
\hline Will you recommend this treatment type to a friend who has MDR TB & 89 & 6 & 1 & 92.7 & 6.3 & 1.0 \\
\hline Do you know about the progress of your treatment? & 65 & 35 & 0 & 63.6 & 36.4 & 0 \\
\hline \multirow[t]{3}{*}{ Patient's satisfaction level } & \multicolumn{3}{|c|}{ Frequency } & \multicolumn{3}{|c|}{ Percentage } \\
\hline & Higl & Medium & Low & High & Medium & Low \\
\hline & 64 & 4 & 28 & 66.7 & 4.2 & 29.2 \\
\hline
\end{tabular}

In table 4 , out of all the patients that participated in the interview and focused group discussion, $81.3 \%$ of patients noted that they felt at home when healthcare workers attended to them. The proportion of patients who felt their privacy was respected were $77.1 \%$. Patients who complained that they did not receive monthly incentives which was to be for transportation \& social support were $38.1 \%$, Patients who received counselling \& health information were $92.7 \%$, patients who understood the health information were $88.5 \%$, patients felt their information were kept confidential, were $85.4 \%$ and $52.1 \%$ did not take waiting time to be too long. Participants that preferred receiving treatment at the treatment centre were $59.4 \%$ while $27.1 \%$ preferred home treatment. Participants who noted that they can recommend the treatment type to a friend who has MDR TB were $92.7 \%$, patients who knew about the progress of their treatment were $63.6 \%$ while $36.4 \%$ did not know.

\section{Patients' satisfaction level (\%)}

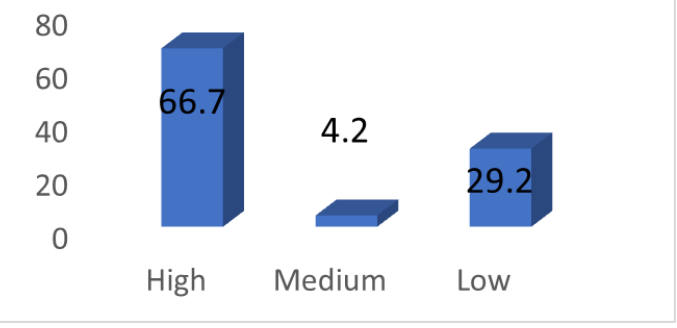

Figure 1. Patients'satisfaction.

About $66.7 \%$ of the patients responded that their satisfaction was high, patients who indicated that their satisfaction was neither high nor low were $4.2 \%$ and $29.2 \%$ indicated a low satisfaction. (figure 1). 


\section{Discussions}

This qualitative study was conducted to identify factors affecting MDR TB treatment adherence. Focus group discussion and structured questionnaires were used to obtain information from patients and healthcare providers. The responses from both patients and healthcare providers revealed that factors related to non-adherence to treatment are multifactorial. However, alcohol addiction, lack of social support, feeling cured, long distance to treatment center, non-payment of stipends/inadequate enablers for patients, stigma \& discrimination, financial difficulty, side effect of treatment, long treatment duration \& high pill burden were the common factors identified to be affecting MDR TB patients' adherence to treatment. Common factors that could affect treatment adherence have been summarized into patient, healthcare provider, socioeconomic and medication related factors. Treatment centre access related or Long distance to treatment centre, inadequate enablers for patients to continue treatment, Lack of social support programs are healthcare provider factor. Medication related factors are side effect of treatment, long treatment duration \& high pill burden Patients related factors are alcohol addiction, careless or nonchalant behavior of not taking drugs as symptoms have been relieved. Financial difficulty, lack of money to manage co-morbidities, stigma and discrimination are socioeconomic factors. The findings of this study is in line with a study done in Plateau State of Nigeria which showed that long distance to treatment sites, poor knowledge of duration of TB treatment, alcohol abuse \& cigarette smoking were major determinants of treatment interruption. This is similar with a study where it was reported that financial constraint through lack of money, transportation cost and food impacts on TB cases' ability to adhere to treatment till the course is completed [3]. Long distance travelling and financial burdens discouraged tuberculosis DOTs treatment initiation and compliance. They further analysed the distances covered by the patients that affected adherence \& found that patients living 0-5 kms away from treatment centre had treatment interruption while many of TB patients who live greater than $5 \mathrm{kms}$ away from the health facility did not adhere. The factor with the highest frequency in this study was long distance to treatment centre. Treatment is free in Nigeria; however transportation is not free. As at the time of this study there were 534 DR TB facility treatment centres \& some community based, yet some patients cover a long distance to access treatment centres. In this study, majority of the patients were found to be unemployed, farmers and petty traders who find it difficult to provide good meals for themselves, so having problem transporting themselves to a distant treatment centre is just an understatement. Alcohol intake had significant association with non-adherence and increased odds of poor treatment outcomes and this could be attributed to loss of thoughts \& control where patients forget counselling \& instructions given by healthcare providers [1-3] Financial difficulty, feeling cured, social support, incentives reported in this study are also in line with previous qualitative studies $[15,2,18,5,13]$. This qualitative study also described the perceptions of MDR TB patients on the care and services they receive from healthcare providers. This study revealed that $81.3 \%$ of patients felt at home when healthcare workers attended to them. This is similar with the qualitative study on Nurses and Patients' experiences on quality of care. In their study, they noted that nurses spend 24 hours/7 days every week with patients, gather large amounts of information about patients and therefore remains the first points of contact for patients [12]. The result of their study showed that direct contact with patients is crucial to building and maintaining a relationship of trust, when these patients feel heard and understood, they consider themselves to be in safe hands and feel at home. Similar findings were also reported in Nigeria and Ethiopia that patients' perceptions on health care provider interaction had a significant influence on positive patient experience and adherence to TB treatment. The proportion of patients who felt their privacy was respected were high (77.1\%) and this is in line with previous studies $[6,7]$. Participants that received incentives were $56.3 \%$ while $38.1 \%$ of patients complained that they did not receive monthly incentives. However, patient-centered approaches, individualized support and monitoring of treatment adherence, use of incentives to continue treatment, and interventions to return patients who abandon treatment have been reported in the literature to improve TB outcomes and directly affect positive experience and perception of patients on care $[10,11]$. Participants that preferred receiving treatment at hospital were $59.4 \%$ while $27.1 \%$ preferred home treatment. People who preferred taking treatment at home were in their continuation phase, who in the course of the focused discussion noted that the frequent visits to treatment centre makes friends and people around to keep asking why they are always visiting hospital. However, majority still preferred being hospitalized.

Findings of this study indicated that the overall patients' level of satisfaction on care was high. This is contrary to the study which revealed a relatively low satisfaction level with the reason that satisfaction is related to changes in quality rather than quality [8]. Changes in quality can lead to changes in satisfaction, as patients notice improvements. However, our finding is in line with findings of patients' satisfaction study done in Tanzania where a high proportion of patients were satisfied with quality of care [9]. Though the satisfaction score was high, some patients reported their dissatisfaction with the inadequate/lack of incentive package. This incentive plays a big role in retaining patients on treatment as some of the patients in this study were not buoyant to have healthy meals and transport themselves to treatment centers. The observed dissatisfaction of patients on incentive underline the importance of hospital management and TB Program to strengthen the incentive package for patients and this should be seen as an area for improvement.

\section{Conclusion}

Some of the identified factors responsible for non-adherence during TB treatment can be potentially addressed through targeted interventions since they were 
categorized under patient-related, health system related, medication related and social related factors. Financial difficulty, lack of social support, treatment side effects and personal factors are associated with non-adherence. From the views of patient and healthcare providers, adherence was conditioned by sociocultural and economic context perspectives. Identifying the majority of factors highlights the areas for opportunities of improvements. Perceptions on care and services received by MDR TB Patients indicated that most patients adhered to their medications, but few who missed treatment appointments were mainly due to perceived wellness, financial difficulty \& lack of incentive and treatment side effect. Patients' satisfaction score was high, however patients recommend that the TB Program should strengthen the incentive and social welfare program for MDR TB cases.

\section{Limitation of This Study}

There is the need to further use a larger dataset to include susceptible population which would give a better insight in assessing factors affecting non-adherence, treatment outcome and MDR TB.

\section{Recommendations}

The findings of this study have shown that:

1. There is need for TB Program to strengthen the system to close up system related factors affecting DR TB services and devise strategies to improve treatment outcome.

2. There is need to strengthen surveillance laying emphasis on disaggregated data by States/Zones, to review gaps and proffer targeted solutions.

\section{Acknowledgements}

Sincere appreciation to participants, healthcare providers of the health facilities, TB Program of the 3 states and others who contributed to the success of this study.

\section{References}

[1] Mekonnen, H. S., \& Azagew, A. W. (2018). Non-adherence to anti-tuberculosis treatment, reasons and associated factors among TB patients attending at Gondar town health centers, Northwest Ethiopia. BMC research notes, 11 (1), 691. https://doi.org/10.1186/s13104-018-3789-4.

[2] Mohammadi, E., Abedi, H. A., Gofranipour, E., Jalali, F \& Kazemnejad, A. (2009). Evaluation of 'partnership care model' in the control of hypertension. International Journal of Nursing Practice, 12, 153-159.

[3] Habteyes Hailu Tola, Azar Tol, Davoud Shojaeizadeh, Gholamreza Garmaroudi (2015). Tuberculosis Treatment Non-Adherence and Lost to Follow Up among TB Patients with or without HIV in Developing Countries: A Systematic Review. Iran J Public Health, Vol. 44, No. 1, pp. 1-11.
[4] Gehre, F., Otu, J., Kendall, L. et al. (2016). The emerging threat of pre-extensively drug-resistant tuberculosis in West Africa: preparing for large-scale tuberculosis research and drug resistance surveillance. BMC Med 14, 160. https://doi.org/10.1186/s12916-016-0704-5.

[5] Sukartini, T., Minarni, I. and Asmoro, C. (2018). Family Support, Self-efficacy, Motivation, and Treatment Adherence in Multidrug-resistant Tuberculosis Patients. In Proceedings of the 9th International Nursing Conference. pages 178-182. DOI: 10.5220/0008322301780182. ISBN: 978-989-758-336-0.

[6] Kalagari Sh, Yazdi K, Mir Karimi Z, Behnampour N (2008). Study of hospitalized patients' views about respecting to the human territory in the 5 Azar hospital in Gorgan Journal of Booie Nursing Faculty of Gorgan. 5: 27-31 [Google Scholar].

[7] Kuzu A, Ergin A, Zencir M. (2006). Patients' awareness of their rights in a developing country. Public Health. 120: 290-6 [PubMed] [Google Scholar].

[8] Khamis, K. and Njau, B. (2014) Patients' level of satisfaction on quality of health care at Mwananyamala hospital in Dar es Salaam, Tanzania. BMC Health Serv Res 14, 400. https://doi.org/10.1186/1472-6963-14-400.

[9] Leshabari M, Muhondwa P, Mwangu M, Mbembati A (2008). Motivation of health care workers in Tanzania; a case study of Muhimbili National Hospital. East Africa Journal of Public Health. 5: 1.

[10] Macq JC, Theobald S, Dick J, Dembele M. (2003). An exploration of the concept of directly observed treatment (DOT) for tuberculosis patients: from a uniform to a customized approach. Int J Tuberc Lung Dis. 7 (2): 103-9. PMID: 12588009.

[11] Pope D. S. and Chaisson R. E, (2003) "TB treatment: as simple as dot?" The International Journal of Tuberculosis and Lung Disease, vol. 7, no. 7, pp. 611-615.

[12] Kieft, R. A., de Brouwer, B. B., Francke, A. L. et al. (2014). How nurses and their work environment affect patient experiences of the quality of care: a qualitative study. BMC Health Serv Res 14, 249 (2014). https://doi.org/10.1186/1472-6963-14-249.

[13] Pradipta IS, Houtsma D, van Boven JFM, Alffenaar JC, Hak E. (2020). Interventions to improve medication adherence in tuberculosis patients: a systematic review of randomized controlled studies. NPJ Prim Care Respir Med. 11; 30 (1): 21. doi: 10.1038/s41533-020-0179-x. PMID: 32393736; PMCID: PMC7214451.

[14] Dahab, M., Charalambous, S., Hamilton, R. et al. "That is why I stopped the ART": Patients' \& providers' perspectives on barriers to and enablers of HIV treatment adherence in a South African workplace programme. BMC Public Health 8, 63 (2008). https://doi.org/10.1186/1471-2458-8-63.

[15] Tola, H. H, Tol, A, Shojaeizadeh, D \& Garmaroudi, G. (2015). Tuberculosis Treatment Non-Adherence and Lost to Follow Up among TB Patients with or without HIV in Developing Countries: A Systematic Review. Iranian journal of public health, 44 (1), 1-11.

[16] Obermeyer, Z., Abbott-Klafter, J. and Murray, C. J. L. (2008). "Has the DOTS strategy improved case finding or treatment success? an empirical assessment," PLoS ONE, vol. 3, no. 3, Article ID e1721. 
[17] Yin, X. Tu, Y. Tong, R. Yang, Y. Wang, S. Cao, Hong Fan, Feng Wang, Yanhong Gong, Ping Yin, Zuxun Lu. (2012). "Development and validation of a tuberculosis medication adherence scale," PLoS ONE, vol. 7, no. 12, Article ID e50328.

[18] World Health Organization. (2011). Global Tuberculosis Control Report. WHO Press, Geneva, Switzerland.

[19] Shibabaw A, Gelaw B, Gebreyes W, Robinson R, Wang S-H, Tessema B (2020) The burden of pre-extensively and extensively drug-resistant tuberculosis among MDR-TB patients in the Amhara region, Ethiopia. PLoS ONE 15 (2): e0229040. https://doi.org/10.1371/journal.pone.0229040.

[20] Chiang Silver and Starke Jeffrey. (2018). Principles and Practice of Pediatric Infectious Diseases (fifth edition). Mycobacterium tuberculosis-134. Pages 790-806.e5.

[21] O'Donnell, M. R., Wolf, A., Werner, L., Horsburgh, C. R., \& Padayatchi, N. (2014). Adherence in the treatment of patients with extensively drug-resistant tuberculosis and HIV in South Africa: a prospective cohort study. Journal of acquired immune deficiency syndromes (1999), $67 \quad$ (1), 22-29. https://doi.org/10.1097/QAI.0000000000000221.

[22] Lackey, N. R. and Wingate, A. L. (1998). The Pilot Study: One Key to Research Success. In P. J. Brink \& M. J. Wood (Eds.). Thousand Oaks, CA: Sage.

[23] Centre for Disease Control (2019) EPI INFO version 7
(StatCalc) sample size calculation for Case control studies using Open Epi.

[24] Oladimeji Olanrewaju, Daniel Adedayo Adeyinka, Lehlogonolo Makola, Kabwebwe Honoré Mitonga, Ekerette Emmanuel Udoh, Boniface Ayanbekongshie Ushie, Kelechi Elizabeth Oladimeji, Jeremiah Chikovore, Musawenkosi Mabaso, Atilola Adeleke, Osman Eltayeb, Oluwatoyin J. Kuye, Gidado Mustapha, Olusoji Mayowa Ige, Joyce Nonhlanhla Mbatha, Jacob Creswell, Joyce M. Tsoka-Gwegweni, Lovett Lawson and Ehimario Uche Igumbor. (2018). Clients' Perception of Quality of Multidrug-Resistant Tuberculosis Treatment and Care in Resource-Limited Setting: Experience from Nigeria. Intechopen: Research and development. 10: 190-206. http://dx.doi.org/10.5772/intechopen.76001.

[25] World Health Organization. (2019). Global Tuberculosis Report 2019; World Health Organization: Geneva.

[26] Olusoji Daniel, Eltayeb Osman, Olanrewaju Oladimeji and Olapade Gbolahan Dairo.(2013). Pre-Extensive Drug Resistant Tuberculosis (Pre-XDR-TB) among MDR-TB Patents in Nigeria. Global Advanced Research Journal of Microbiology. Vol. 2 (2) pp. 1-5. http://garj.org/garjm/index.htm.

[27] Onyedum C. C., Alobu I., Ukwaja K. N. (2017). Prevalence of drug-resistant tuberculosis in Nigeria: A systematic review and meta-analysis. PLoS ONE 12 (7): e0180996. https:// doi. org/ 10. 1371 / journal.pone.0180996. 